\title{
Multi-component layout design with coupled shape and topology optimization
}

\author{
J.H. Zhu ${ }^{1,2, a}$, W.H. Zhang ${ }^{1}$ and P. Beckers ${ }^{2}$ \\ 1 Sino-French Laboratory of Concurrent Engineering, Northwestern Polytechnical University 710072 Xi'an, Shaanxi, P.R. China \\ 2 LTAS - Infographie, Université de Liège, 4000 Liège, Belgium
}

Received 30 March 2008, Accepted 15 Juin 2008

\begin{abstract}
A Coupled Shape and Topology Optimization (CSTO) method is proposed here to deal with the layout design of the multi-component system. Considering a complex packing system for which several components will be placed in a container of specific shape, the aim of the design procedure is to find the optimal location and orientation of each component as well as the configuration of the structure that supports and interconnects the components. Compared with existing packing design approaches, two significant improvements are made in the CSTO method. On the one hand, a new Finite-circle Method (FCM) is used here to discretize boundaries of all components and the container into a number of circumcircles. Hence geometric constraints can be suitably modelled to avoid the overlap among the components as well as the overlap between the components and the container contour. Besides, the FCM approximation is also convenient to deal with components and the container with concave or some other complex shapes. On the other hand, the design procedure is able to take into account the mechanical performances of the structural system. Here, the location and the orientation of the components will be updated to improve the system rigidity by using shape optimization procedure. Meanwhile, the optimal material layout of the supporting structure in the design domain is designed by topology optimization. Due to the iterative movement of components, the technique of the embedded mesh is used to update the local FE mesh around each component in the design domain and pseudo density design variables assigned to density points instead of finite elements will be used to follow such a FE mesh variation. Several design problems are tested in this paper, and numerical results show the proposed CSTO method extends the actual concept of topology optimization and is efficient to generate reasonable design patterns.
\end{abstract}

Key words: Multi-component system; shape and topology optimization; finite-circle method; concave shape; density points; embedded mesh.

\section{Introduction}

Structural optimization and its industrial applications are always active research topics in engineering community covering aerospace, marine and mechanical systems, etc. Among others, structural topology optimization is recognized increasingly as one of the powerful tools supporting practical structural designs. As we can see, most of the industrial products can be considered to be multicomponent systems made up of a container, i.e., a design domain and a number of components and structures to support and interconnect the container and components. In this paper, two basic problems involved in the design of a multi-component system are discussed.

On the one hand, the position and orientation of each component in the design domain should be designed. This is a kind of packing optimization problem. Packing problem or configuration design is still a CAD-based design with the compactness, position of gravity centre, etc. to

\footnotetext{
${ }^{a}$ Corresponding author: jh.zhu_fea@hotmail.com
}

be the design objective (see $[1,2])$. The components placement in the design domain can be treated as a shape optimization problem in which component locations and orientations are defined as the design variables. Among others, one of the key difficulties lies in that the geometry constraints have to be properly specified in order to avoid the components overlap and their overlap with the design domain boundary. However, varieties of component shapes and design domain boundaries will lead to nonlinearity and even discontinuity of the constraint functions, especially when the components or the design domain boundary have complex, concave or even 3D shapes, that limit the application of the gradient based optimization algorithms in the traditional formulation of packing problems. Therefore, non-deterministic computational approaches such as Genetic Algorithms (GA), Simulated Annealing and some extended patterns are mostly used in packing optimization problem. Recently, Zhang and Zhu [3] propose a new Finite-Circle Method (FCM). The idea is to replace approximately the exact shape of 

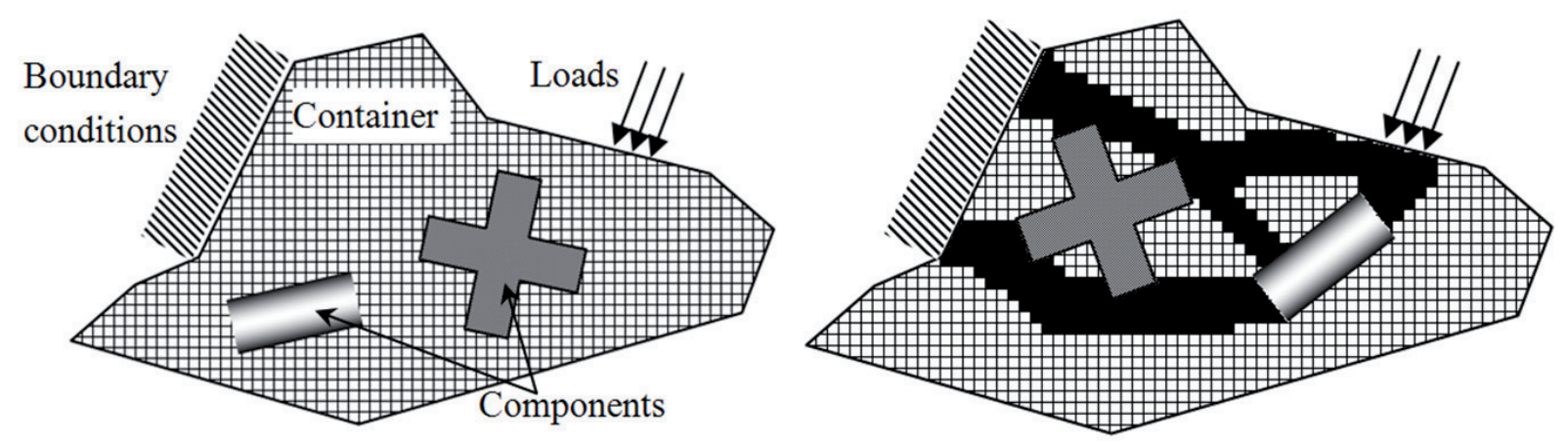

Fig. 1. An illustrative multi-component design problem.

each component with a family of circum-circles. Thus, the complex overlap constraints between components can be approximated into distance constraints between circumcircle centres themselves. As a result, constraint functions are kept to be continuous, differentiable and beneficial in sensitivity analysis and application of gradient based algorithms.

On the other hand, the system performances of embedded objects are the primary design objectives to be optimized. With this idea in mind, the design of the supporting structures connecting the components under specific loads and boundary conditions can be stated as a topology optimization problem. In the past one or two decades, standard topology optimization methods are developed to find optimal material layout for a single prescribed design domain with predefined loads and boundary conditions (see [4]). Meanwhile, effective approaches like homogenization based method [5,6], SIMP model (Solid Isotropic Material with Penalization) [7,8], evolutionary method [9], etc. have been proposed so far. Later on, more extended approaches are proposed to solve some complex problems such as microstructures design (see [10,11]) and design-dependent loads $[12,13]$. Moreover, the concept of topology design domain is also extended to design structural supports and joints modeled with spring elements. Some numerical examples of the latter were presented by Jiang and Chirehdast [14], Buhl [15], Zhu and Zhang [16] to solve structural stiffness, compliant mechanism and natural frequency problems. By constraining the material volume and the number of interconnections, Chickermane and Gea [17], Qian and Ananthasuresh [18] assigned each component and their joints as sub-design domains in topology optimization, respectively.

In fact, both of the above two aspects have to be carried out simultaneously in a multi-component system design. Compared to the standard topology optimization procedure in which topology design variables are assigned to elements of a fixed mesh, difficulties raised here are how to define topology design variables for a moving mesh due to the components placement in shape design iteration. Qian and Ananthasuresh [18] presented a compromised approach, in which the exact geometry of each component is not included but simulated with a predefined material interpolation model, i.e., the geometrical movement of a component is actually simulated as a physical variation of the material properties, while the supporting structure is designed with the standard topology optimization.

In this paper, an alternative method named CSTO is proposed. The exact geometry contour of each component will be modeled and embedded in the design domain. By means of the FCM method, the components layout is optimized with standard sensitivity-based shape optimization procedure while the supporting structures are designed with a new topology optimization approach. An illustrative multi-component system design problem is shown in Figure 1. By assigning the density variables to the predefined density points rather than finite elements, this approach doesn't require a fixed FE mesh. Relevant examples with components embedded in the design domain will be solved using the CSTO in the present paper.

\section{Formulation of finite-circle method}

In existing packing optimization approaches, different functions are used to describe each particular component with different shapes. Few of these functions are however differentiable and lead to the difficulties in sensitivity analysis. The FCM proposed by Zhang and Zhu [3] is a general method for packing optimization. The complex geometry of each component is transformed into a family of circum-circles so that the overlap between two components is avoided by constraining the distance between two related circles, which is a simple and differentiable function. Here, this method is improved to take into account more complex situation where the boundary of the design domain is concave.

\subsection{Components approximation}

As shown in Figure 2, $\Gamma_{1}$ and $\Gamma_{2}$ are the domains occupied by the 1 st and 2 nd components respectively. One design constraint should be defined to avoid the overlap of them

$$
\Gamma_{1}\left(x_{1}, y_{1}, \theta_{1}\right) \cap \Gamma_{2}\left(x_{2}, y_{2}, \theta_{2}\right)=\emptyset .
$$

Unfortunately, this kind of description is unable to directly be applied in our optimization model. By FCM, 

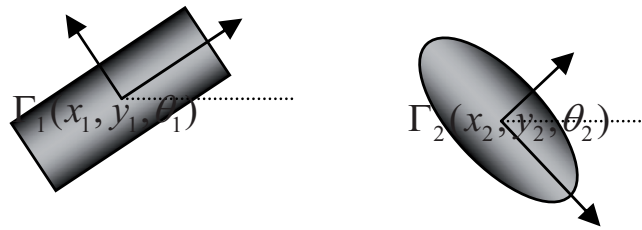

Fig. 2. Two components without any approximation.

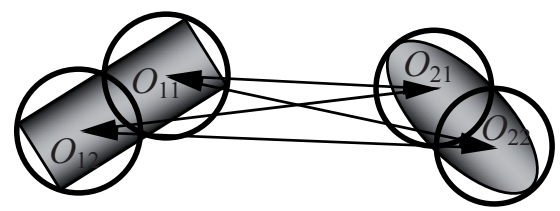

Fig. 3. Simplified geometry constraints with FCM.
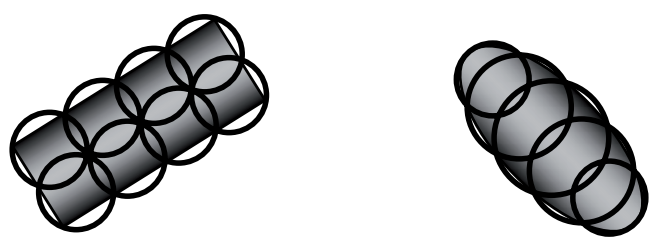

Fig. 4. A refined approximation with more circum-circles.

circum-circles are used to approximate each component as shown in Figure 3.

To avoid the overlap of the components, circles belonging to one component are constrained not to overlap with any circle of the other component. In Figure 3, two circles are attached to each component and the design constraints can be written as

$$
\begin{aligned}
& \left\|O_{11} O_{21}\right\| \geqslant R_{11}+R_{21}, \quad\left\|O_{11} O_{22}\right\| \geqslant R_{11}+R_{22} \\
& \left\|O_{12} O_{21}\right\| \geqslant R_{12}+R_{21},\left\|O_{12} O_{22}\right\| \geqslant R_{12}+R_{22}
\end{aligned}
$$

where $O_{11}$ denotes the centre of the 1st circle belonging to the 1st component, whose coordinates can be calculated by the location and orientation of the component. $R_{11}$ is the radius of this circle. Now, the complex geometry constraint of equation (1) is expanded to several constraints of simple form shown in equation (2) in a systematic way.

In most cases except that the component is originally circular or holds some other particular shapes, the precision of the approximation can be improved by increase the number of circum-circles as shown in Figure 4. This is just like the refinement scheme of Finite Element Method. In Figure 4, we can find the approximation is more precise but it leads to 40 design constraints of equation (2). Similarly, for a 3D problem, the circum-circles will be upgraded to spheres for the components approximation.

\subsection{Design domain boundary approximation}

Although the components have been described by families of circum-circles, additional geometry constraints should be defined to ensure all components are completely lo-

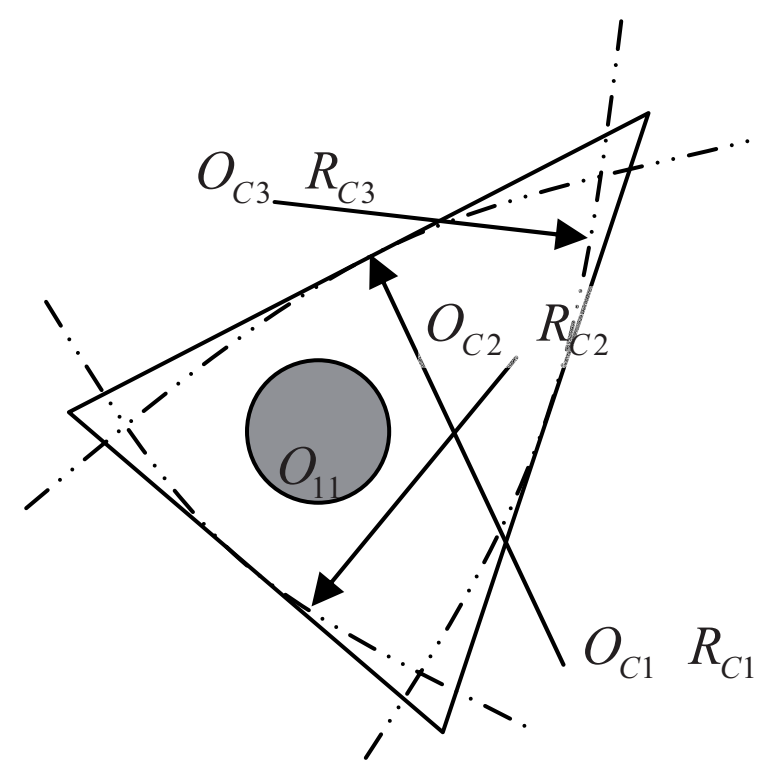

Fig. 5. Approximation of a convex design domain.

cated inside the design domain. Namely,

$$
\begin{aligned}
& \Gamma_{1}\left(x_{1}, y_{1}, \theta_{1}\right) \subset \Gamma_{D} \\
& \Gamma_{2}\left(x_{2}, y_{2}, \theta_{2}\right) \subset \Gamma_{D}
\end{aligned}
$$

where $\Gamma_{D}$ is the area occupied by the global design domain Regarding components shown in Figure 2 as an example, equation (3) can be transformed to

$$
\begin{aligned}
& \Gamma_{11} \subset \Gamma_{D}, \Gamma_{12} \subset \Gamma_{D} \\
& \Gamma_{21} \subset \Gamma_{D}, \Gamma_{22} \subset \Gamma_{D}
\end{aligned}
$$

where $\Gamma_{11}$ denotes the area taken by the circle $O_{11}$. Without the loss of generality, let us consider the circle $O_{11}$.

As shown in Figure 5, suppose the circle $O_{11}$ is located in a triangle design domain whose contour is modelled as several circular arcs. Now the constraint becomes

$$
\begin{aligned}
& \left\|O_{11} O_{C 1}\right\| \geqslant R_{C 1}-R_{11} \\
& \left\|O_{11} O_{C 2}\right\| \geqslant R_{C 2}-R_{11} \\
& \left\|O_{11} O_{C 3}\right\| \geqslant R_{C 3}-R_{11} .
\end{aligned}
$$

The precision of the contour approximation can also be improved by adjusting centre positions and the radiuses of big circular arcs.

However, for a concave design domain shown in Figure 6, an improvement of FCM is needed. To this end, the concave part of the design domain is filled and considered as a fictive fixed component $\Gamma_{F}$. In this way, the design domain is transformed into a convex one and this method can be directly applied for general cases. Only more overlap constraints related to equation (6) are added to equation (5).

$$
\Gamma_{11} \cap \Gamma_{F}=\emptyset \text {. }
$$

\subsection{A packing example}

A compact packing design example is tested here to show how the FCM works. In Figure 7, 6 equilateral triangles 


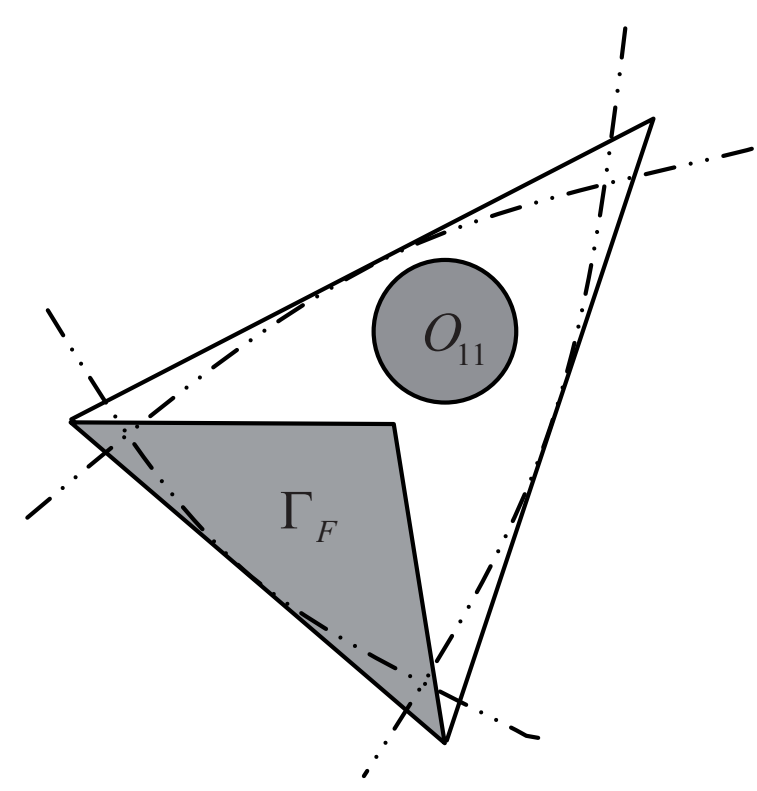

Fig. 6. Approximation of a concave design domain.

are considered to be the components. And one circle is defined as the boundary of packing domain. Each triangle will be approximated with 9 small circles.

The design purpose is to find the layout of 6 components and a packing domain of minimum area being able to contain them without overlap. The original configuration is shown in Figure 8. By using the gradient based GCM algorithm of BOSS-Quattro ${ }^{T M}$ software [19], this problem costs 9 iterations to reach the convergence. The design iteration and the final configuration are shown in Figure 9.

In the final configuration, 6 equilateral triangles are arranged compactly as an equilateral hexagon. It should be mentioned that this configuration is a feasible compact design because of the conservative circum-circle approximation.

\section{Coupled shape and topology optimization}

As the mechanical performances are included in the multicomponent system optimization, the design procedure is much more complicated than a pure packing design. It is concerned with an integrated design of the packing and supporting structures and can be formulated as a CSTO problem.

\subsection{Density points and embedded mesh technique}

For a CSTO problem, the difficulty lies in that when the shape parameters are changed, i.e., the components translate or rotate in the design domain, the FE mesh should be updated simultaneously. As a result, the pseudodensities as used before in topology optimization can no longer be assigned to fixed elements. Here, the concept of the density points is presented. As shown in Figure 10a,

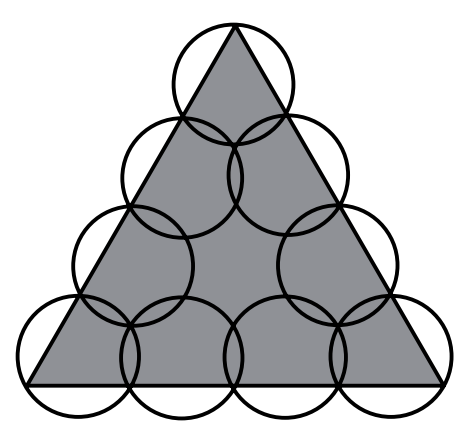

Fig. 7. Equilateral triangle component approximated with 9 circles.

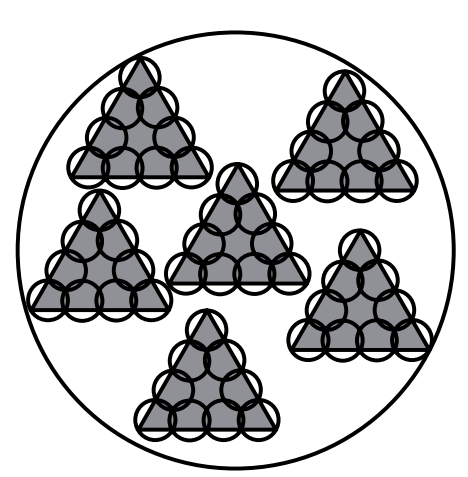

Fig. 8. The original configuration.

density points are prescribed in a design domain. Pseudodensity values (0-1) of design variables are attached to these points that remain unchanged during the optimization. For each finite element discretizing the design domain, the nearest density point will be identified and the pseudo-density value will be attributed correspondingly to the element. As illustrated in Figure 10b, the pseudodensity value of density point 1 will be attributed to the considered element as the distance from this density point to the centroid of the element is the shortest. Hence, one density point may dominate a group of local elements.

In summary, the procedure is as follows. Firstly, the whole design domain is discretized into finite elements. Such a mesh is here referred to as basic mesh whose centroids are used to define positions of density points as shown in Figure 11a. Suppose components are meshed independently. As they are placed in the design domain, an embedded mesh consisting of the basic mesh and component meshes is then created automatically by local mesh merging and smoothing as shown in Figure 11b. At this rate, when positions and orientations of the components change in the optimization process, only local mesh merging and smoothing are needed while most elements of the basic mesh remain unchanged.

\subsection{Optimization problem formulation}

Consider the stiffness of a system of $m$ components as the objective function. The simultaneous design of the 


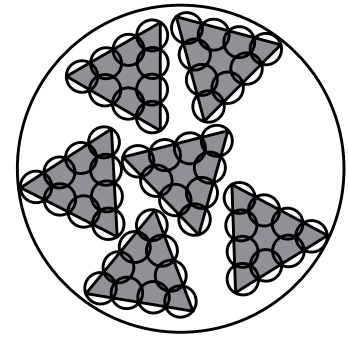

(a) Iteration 3

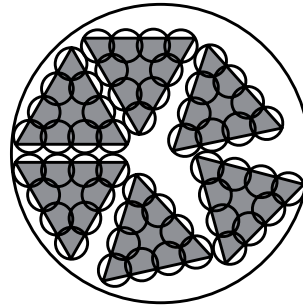

(b) Iteration 5

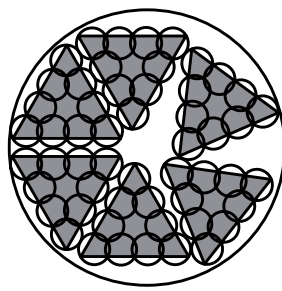

(c) Iteration 7

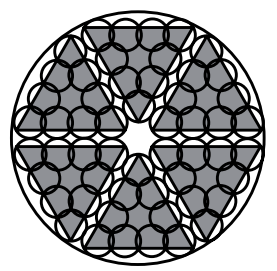

(d) Final configuration

Fig. 9. Design iteration and the packing result.

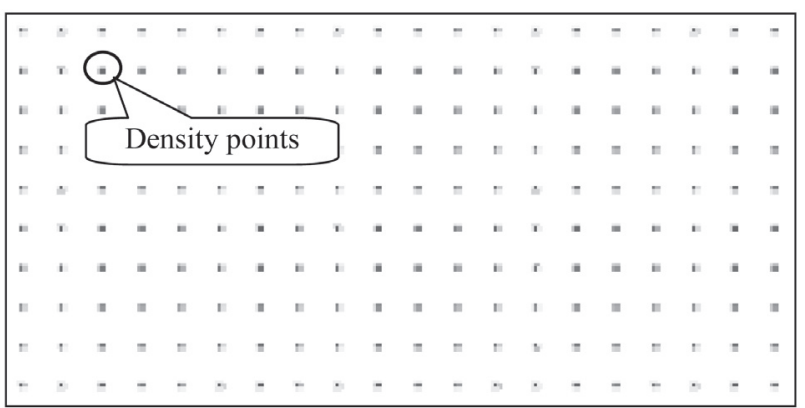

(a) The orderly placed density points

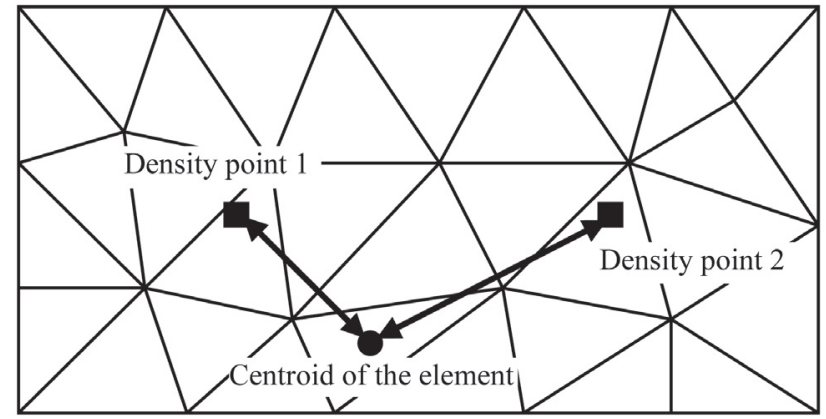

(b) The density points and surrounding elements

Fig. 10. Introduction of the density points.

components packing and supporting structures subject to the volume and geometry constraints can be expressed as

Find $: \quad \boldsymbol{\eta}=\left\{\eta_{1}, \eta_{2}, \ldots, \eta_{n}\right\} \quad \boldsymbol{S}=\left\{\begin{array}{ccc}x_{1} & y_{1} & \theta_{1} \\ x_{2} & y_{2} & \theta_{2} \\ & \vdots & \\ x_{m} & y_{m} & \theta_{m}\end{array}\right\}$

$\min : \quad C=\frac{1}{2} \boldsymbol{u}^{T} \boldsymbol{K} \boldsymbol{u}$

s.t. : $\quad V \leqslant V^{u}$

$0<\eta^{l} \leqslant \eta_{i} \leqslant 1(i=1,2, \ldots, n)$

$\Gamma_{j}\left(x_{j}, y_{j}, \theta_{j}\right) \cap \Gamma_{k}\left(x_{k}, y_{k}, \theta_{k}\right)=\emptyset$

$\Gamma_{j}\left(x_{j}, y_{j}, \theta_{j}\right) \subset \Gamma_{D}$

$j \neq k(j=1,2, \ldots, m k=1,2, \ldots, m)$

where $\eta_{i}$ is the pseudo-density of the $i$ th density point. $\eta^{l}$ is the lower bound of the density variables, which is set to be 0.001. $S$ is the shape design variable matrix

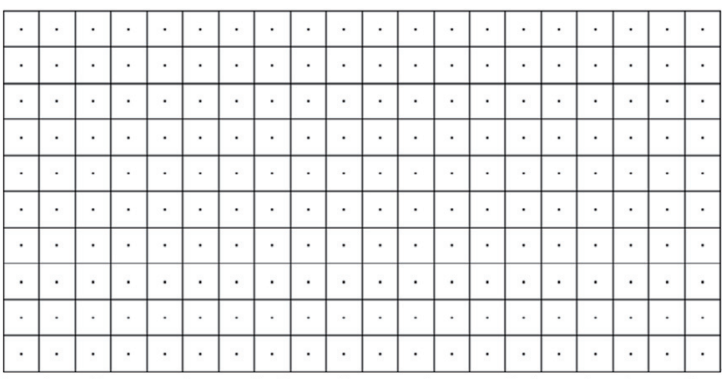

(a) The basic mesh and density points of the design domain

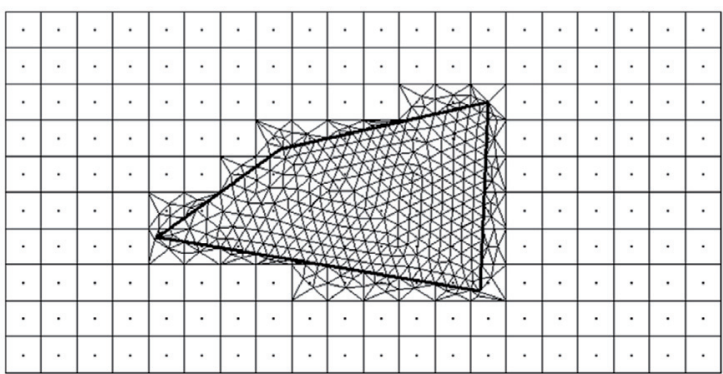

(b) The embedded mesh

Fig. 11. Modeling of the basic mesh and embedded mesh.

consisting of all the component location and orientation parameters. $C$ is the global strain energy of the structure calculated by the nodal displacement $\boldsymbol{u}$ and the global stiffness matrix $\boldsymbol{K} . V$ and $V^{u}$ are the structural volume and its upper bound, respectively.

Sensitivity analysis consists in evaluating the gradient of the global strain energy with respect to the shape parameters of the components and the pseudo-density variables.

In FE method, the static equation of the structure is represented by

$$
f=K u
$$

where $f$ is the load vector. The gradient of the shape parameter is derived with a finite difference approach. Suppose $s_{j}$ is one of three shape parameters $x_{j}, y_{j}$ and $\theta_{j}$ with the chosen perturbed step size to be

$$
\Delta s_{j}=10^{-5}\left|s_{j}^{u}-s_{j}^{l}\right|
$$


where $s_{j}^{u}$ and $s_{j}^{l}$ are the upper and lower bounds of $s_{j}$, respectively. While the derivative of the global strain energy with respect to $s_{j}$ can be approximated as

$$
\frac{\partial C}{\partial s_{j}} \approx \frac{\Delta C}{\Delta s_{j}}
$$

Moreover, sensitivity with respect to the pseudo-density variables is derived in a standard analytical scheme. Suppose $\eta_{i}$ dominates llocal elements, the element stiffness matrix attached to $\eta_{i}$ can be written as

$$
\boldsymbol{K}_{i e}=\eta_{i}^{p} \boldsymbol{K}_{i e}^{0}(e=1,2, \ldots, l)
$$

where $p$ is the penalty factor set to be 3 in this paper. $\boldsymbol{K}_{i e}^{0}$ is the element stiffness matrix when the element is full of solid material. Likewise, the sensitivity is also formulated by differentiating the static equation.

$$
\frac{\partial \boldsymbol{K}}{\partial \eta_{i}} \boldsymbol{u}+\boldsymbol{K} \frac{\partial \boldsymbol{u}}{\partial \eta_{i}}=0
$$

The derivative can be expressed as

$$
\frac{\partial C}{\partial \eta_{i}}=\frac{1}{2} \boldsymbol{f}^{T} \frac{\partial \boldsymbol{u}}{\partial \eta_{i}}=\frac{1}{2} \boldsymbol{u}^{T} \boldsymbol{K} \frac{\partial \boldsymbol{u}}{\partial \eta_{i}}=-\frac{1}{2} \boldsymbol{u}^{T} \frac{\partial \boldsymbol{K}}{\partial \eta_{i}} \boldsymbol{u} .
$$

Since only $\boldsymbol{K}_{i e}(e=1,2, \ldots, l)$ are related to $\eta_{i}$, this equation can be further developed as

$$
\begin{aligned}
\frac{\partial C}{\partial \eta_{i}} & =-\frac{1}{2} \boldsymbol{u}^{T} \frac{\partial\left(\sum_{e=1}^{l} \boldsymbol{K}_{i e}\right)}{\partial \eta_{i}} \boldsymbol{u} \\
& =-\frac{p}{2} \boldsymbol{u}^{T} \frac{\sum_{e=1}^{l} \boldsymbol{K}_{i e}}{\eta_{i}} \boldsymbol{u}=-\frac{p}{\eta_{i}} \sum_{e=1}^{l} C_{i e}
\end{aligned}
$$

where $C_{i e}$ is the strain energy of the eth element.

\subsection{Multi-component system design examples}

Some problems with solid components are tested in this section to illustrate how to optimize the CSTO design of the component packing and supporting structures of a complex multi-component system. The material properties are as follows.

Supporting structure: Elastic modulus $E=1$ pa; Poisson's ratio $\nu=0.3$;

Solid component: Elastic modulus $E=3$ pa; Poisson's ratio $\nu=0.3$

The latter is stiffer than the supporting structure.

The first design domain as shown in Figure 12 is a plate with a size of $6 \mathrm{~m} \times 12 \mathrm{~m}$. The basic mesh is divided into $30 \times 60$ finite elements. Suppose the density points are defined at the centroids of the basic elements. The load and boundary conditions are also described in Figure 12 .

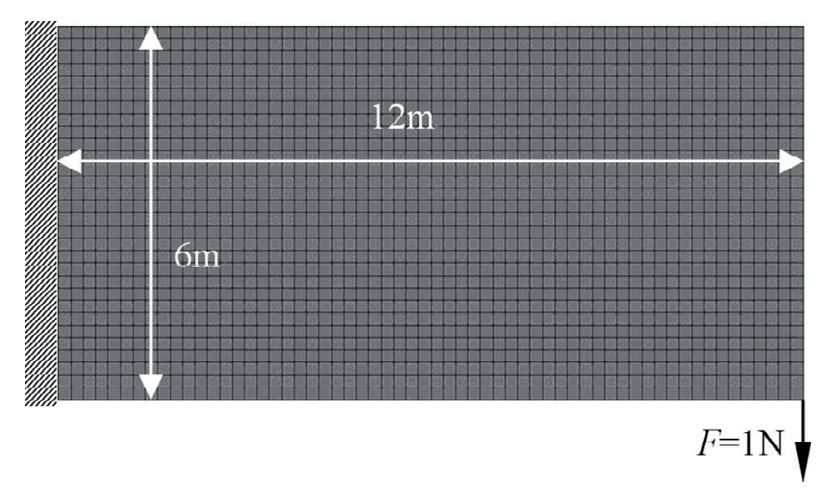

Fig. 12. Design domain and basic mesh of the plate.

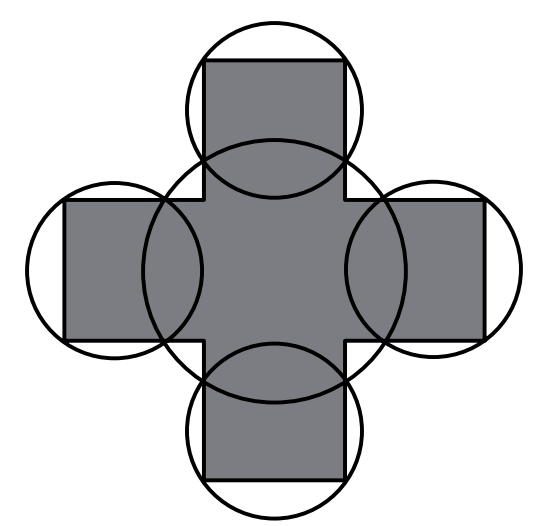

Fig. 13. Component and its circles.

As shown in Figure 13, one solid component will be approximated with 5 circles. The component is initially placed at the center of the plate. By minimizing the strain energy and constraining the volume of the supporting structures to $50 \%$ of the total, this problem is carried out with CSTO. The optimization is converged at the $32 \mathrm{nd}$ iteration. Several iterations and the final configuration are shown in Figure 14, where the component is located as a part of the structure.

The second design domain and its dimension are shown in Figure 15a. An L shape beam is modeled with its upper side fixed and one concentrated force is assigned at the right side. The basic mesh, its density points and the FCM boundary approximation are all presented in Figure 15b. For the concave part of the design domain, a fictive fixed triangle component is defined and approximated with 3 circles.

A solid component as shown in Figure 13 will be located in the design domain. It is approximated with 4 circles and shown in Figures 16a and 16b, respectively.

By minimizing the strain energy and constraining the volume of the supporting structures to $50 \%$ of the total volume, this problem is solved. Several iterations of the configuration patterns and the convergence of the design objective are shown in Figure 17. The component is finally located as a part of the structure after 58 iterations.

The last case is about two identical components illustrated in Figure 16 in the same design domain. Figure 18 shows the iteration histories. It can also found that both 


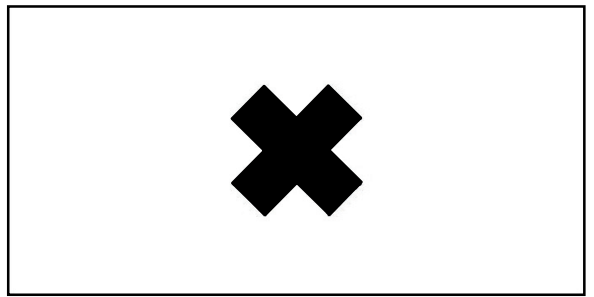

(a) Initial configuration

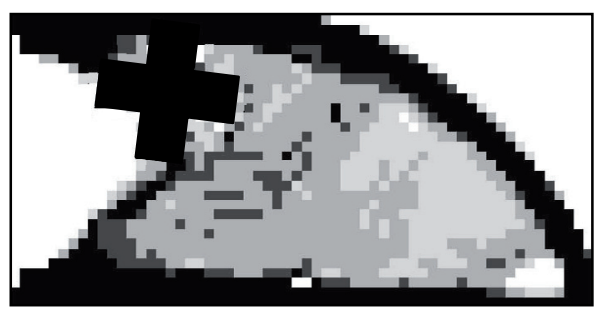

(c) Iteration 25

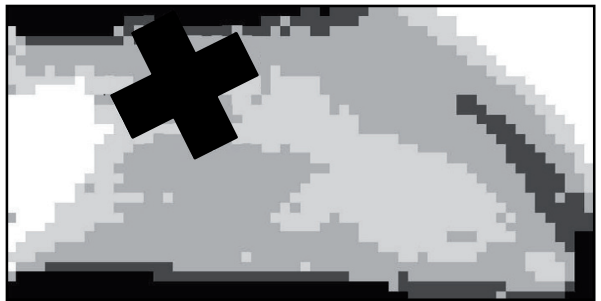

(b) Iteration 5

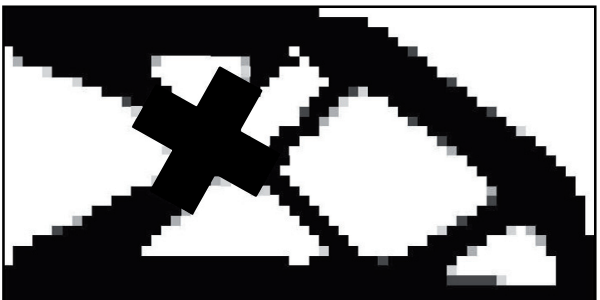

(d) Final configuration

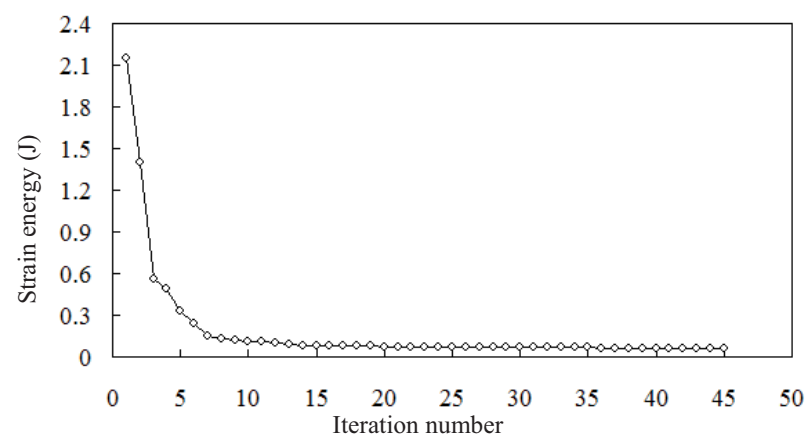

(e) The convergence history of the objective function

Fig. 14. Design iteration and the final configuration.

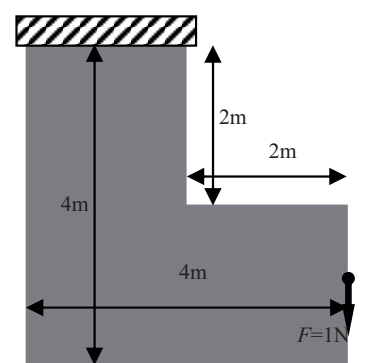

(a) Dimension, load and boundary conditions

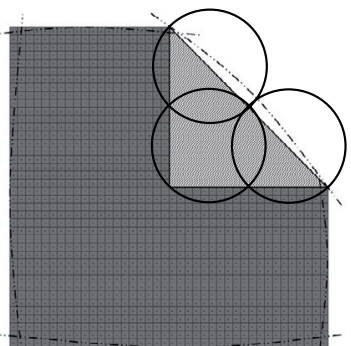

(b) Basic mesh, density points and FCM approximation

Fig. 15. The design domain.

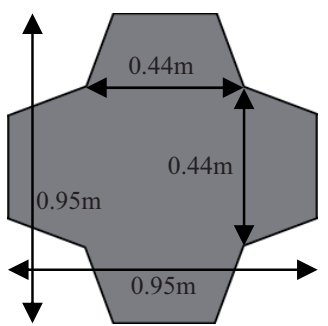

(a) Dimension

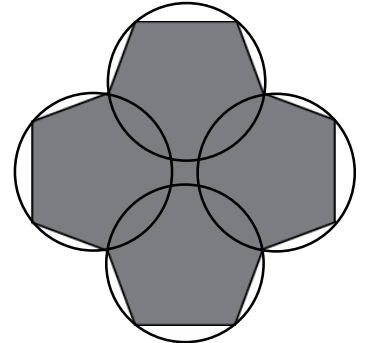

(b) FCM approximation

Fig. 16. The component. 


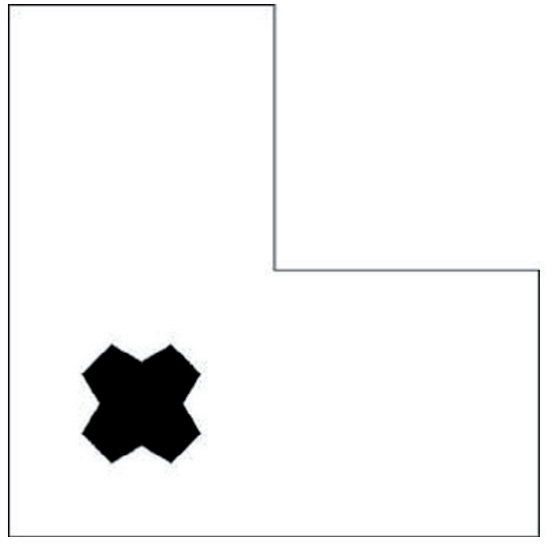

(a) Initial configuration

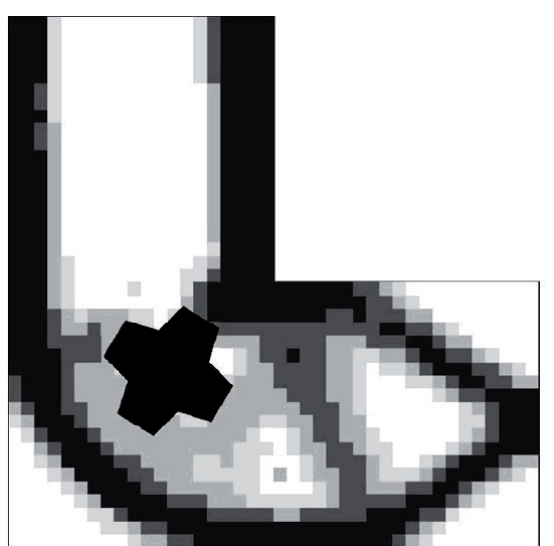

(c) Iteration 25

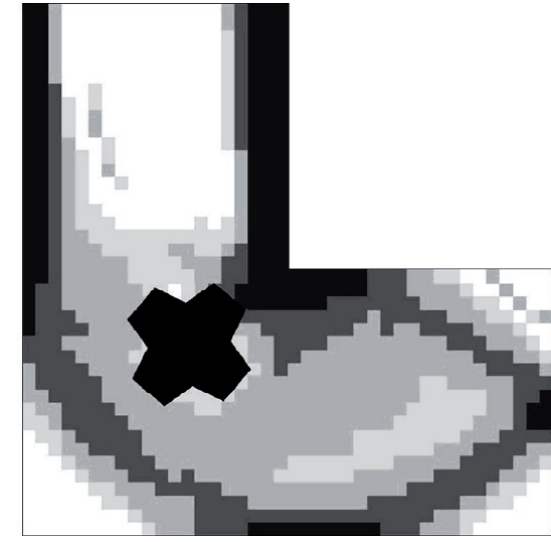

(b) Iteration 10

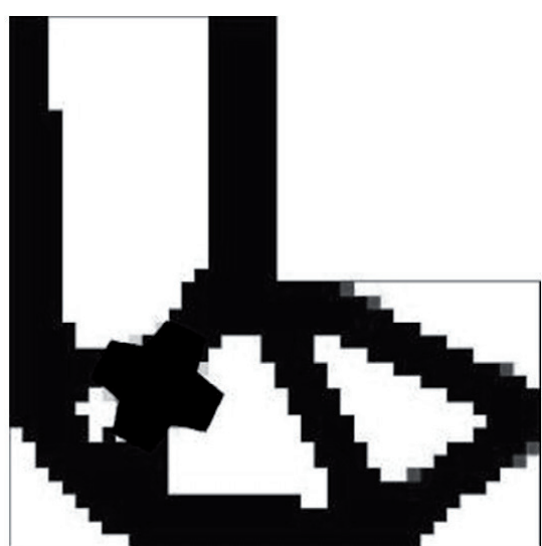

(d) Final configuration

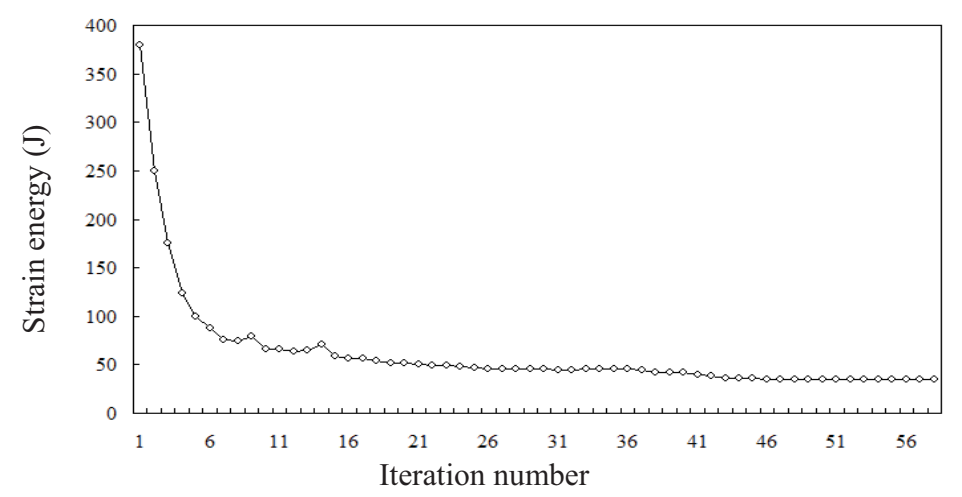

(e) The convergence history of the objective function

Fig. 17. The design process with one solid component.

components are now integrated as parts of the structure and demonstrate their resistance for the rigidity maximization of the system.

\section{Conclusion}

In this paper, components and their supporting structures are simultaneously designed with a proposed CSTO method that integrates the FCM, density point and embedded mesh techniques. The FCM aims at simplifying the complex overlap constraints and improvements are proposed for the concave case. The technique of density points is used to avoid the confliction between shape optimization and topology optimization. Meanwhile, this approach together with the embedded mesh technique can reduce the computing cost of the remeshing procedure. Two examples including concave design domain are solved. Numerical results show that CSTO is efficient to achieve reasonable design configurations. 


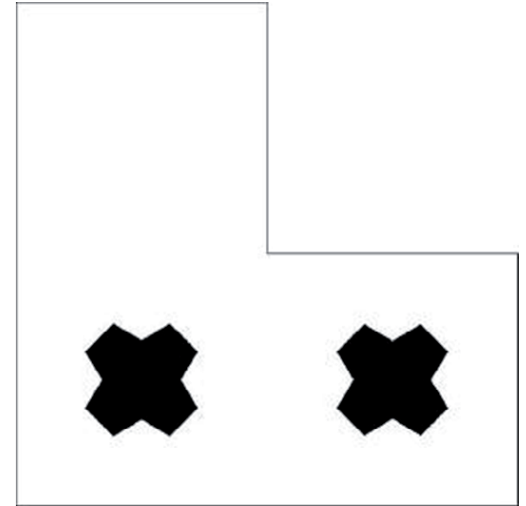

(a) Initial configuration

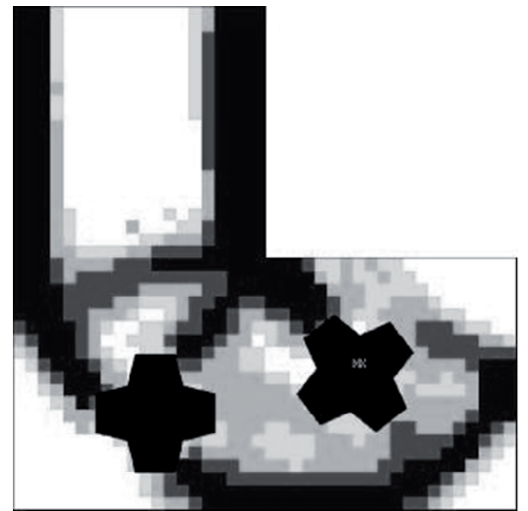

(c) Iteration 25

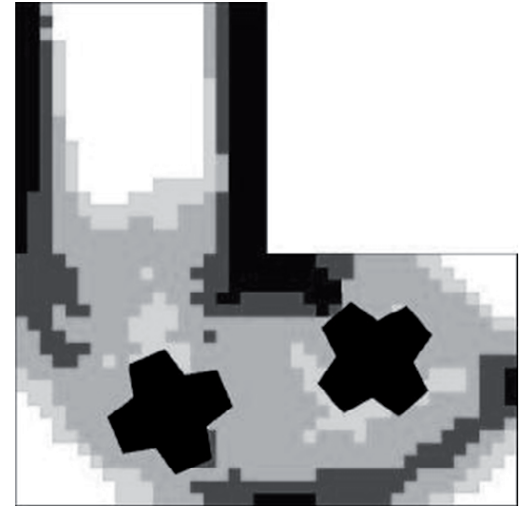

(b) Iteration 10

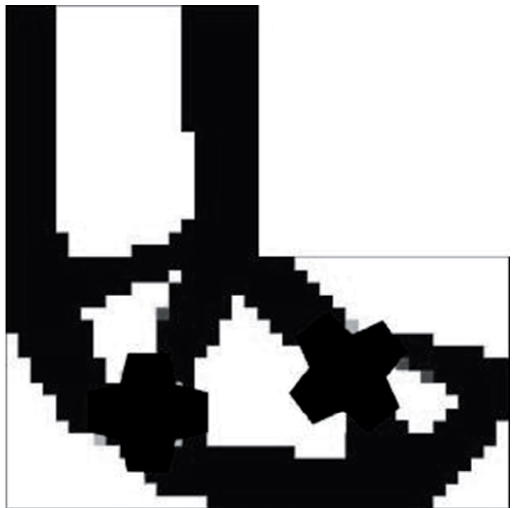

(d) Final configuration

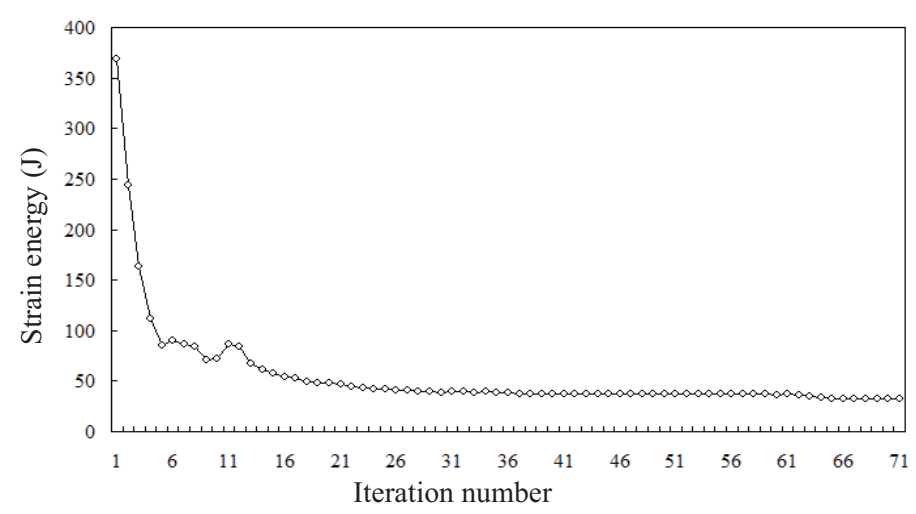

(e) The convergence history of the objective function

Fig. 18. The design process with two solid components.

\section{Acknowledgements}

This paper was published in ASMDO proceeding of First International conference on multidisciplinary optimization and Applications, April 17-20, 2007, ISBN 978-2-7598-0023-0.

This work is supported by the National Natural Science Foundation of China (10676028, 90405016), Aeronautical Science Foundation of China (2006ZA53006, 04B53080), 973 Program (2006CB601205), 863 Project (2006AA04Z122), 111 Project (B07050), Shaanxi Province Science \& Technology Project (2006K05-G25) and Doctorate Foundation of Northwestern Polytechnical University (CX200508).

\section{References}

1. J. Cagan, K. Shimada, S. Yin, Computer-Aided Design 34, 597 (2002)

2. V.Y. Blouin, Y. Miao, X. Zhou, G.M. Fadel, An assessment of configuration design methodologies, in $10 \mathrm{th}$ AIAA/ISSMO (New York, 2004)

3. W.H. Zhang, J.H. Zhu, A new finite-circle family method for optimal multi-component packing design, in WCCM VII (Los Angeles, 2006)

4. M.P. Bendsøe, O. Sigmund, Topology optimization: theory, method and application (Springer, Berlin, Heidelberg New York, 2003) 
5. M.P. Bendsøe, N. Kikuchi, Compt. Methods Appl. Mech. Eng. 71, 197 (1988)

6. G. Allaire, F. Jouve, H. Maillot, Struct. Multidisciplinary Optim. 28, 87 (2004)

7. M.P. Bendsøe, Struct. Optim. 10, 193 (1989)

8. M. Zhou, G.I.N. Rozvany, Comput. Methods Appl. Mech. Eng. 89, 197 (1991)

9. Y.M. Xie, G.P. Steven, Evolutionary structural optimization (Springer-Verlag, Berlin, 1997)

10. O. Sigmund, S. Torquato, J. Mech. Phys. Solids 45, 1037 (1997)

11. W.H. Zhang, S.P. Sun, Internat. J. Numer. Methods Eng. 68, 993 (2006)

12. B.C. Chen, N. Kikuchi, Finite Elements in Analysis and Design 37, 57 (2001)
13. M. Bruyneel, P. Duysinx, Struct. Multidisciplinary Optim. 29, 245 (2005)

14. T. Jiang, M. Chirehdast, J. Mechanical Design 119, 40 (1997)

15. T. Buhl, Struct. Multidisciplinary Optim. 23, 336 (2001)

16. J.H. Zhu, W.H. Zhang, Struct. Multidisciplinary Optim. 31, 462 (2006)

17. H. Chickermane, H.C. Gea, Eng. Comput. 13, 234 (1997)

18. Z.Y. Qian, G.K. Ananthasuresh, Mech. Based Design Struct. Mach. 32, 165 (2004)

19. A. Remouchamps, Y. Radovcic, Boss-Quattro Documents (SAMTECH Inc., 2005) 